Commun. Fac Sci. Univ. Ank. Ser. A1 Math. Stat.

Volume 69, Number 1, Pages 320-335 (2020)

DOI: $10.31801 /$ cfsuasmas. 556898

ISSN 1303-5991 E-ISSN 2618-6470

http://communications.science.ankara.edu.tr/index.php?series=A1

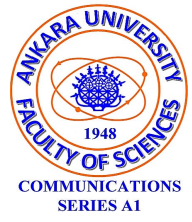

\title{
PRO-C COMPLETIONS OF CROSSED SQUARES OF COMMUTATIVE ALGEBRAS
}

\author{
HATICE GÜLSÜN AKAY
}

\begin{abstract}
In this paper we give the explicit construction of a pro- $\mathcal{C}$ completion functor which is defined in the category of crossed squares of commutative algebras. Afterwards, we study some functorial properties of this pro- $\mathcal{C}$ completion process.
\end{abstract}

\section{INTRODUCTION}

A profinite group [9] occurs in a wide range of problems related to number theory, commutative algebra, algebraic geometry and algebraic topology. Although the category of profinite groups forms a natural extension of the category of finite groups, it carries a richer structure. Because it has some categorical objects and constructions which do not exist in finite case; e.g. projective limits and free products. The existence of such constructions in extended category leads to the definition of profinite analogues of the usual constructions of combinatorial group theory, such as free groups and presentations of group by generators and relations.

The theory of crossed modules [10] plays an important role in combinatorial and cohomological group theory. Profinite crossed modules are introduced in [7. They examined the pro- $\mathcal{C}$ completion of crossed modules for a full class of finite groups $\mathcal{C}$. The crossed square version of this completion process is given in 3 . The definition of crossed modules over a commutative algebra is given in [8]; also see [5] for the general case. Crossed squares in the category of commutative algebras studied in [1, 4.

\section{Preliminaries}

In this paper, $\mathrm{k}$ will be a fixed commutative ring with $1 \neq 0$ for abstract cases and $\mathrm{k}$ will be a fixed commutative profinite ring with $1 \neq 0$ for topological cases.

Received by the editors: April 22, 2019; Accepted: October 17, 2019.

2010 Mathematics Subject Classification. 18G30, 18G55.

Key words and phrases. Crossed square, pro- $\mathcal{C}$ crossed square, pro- $\mathcal{C}$ completion. 
All k-algebras will be commutative and associative. $\mathcal{C}$ will denote a class of finite kalgebras which is closed under the formation of subalgebras, homomorphic images, finite products and which contains at least one non-trivial algebra. Pro- $\mathcal{C}$ algebras are profinite algebras whose finite quotients are in $\mathcal{C}$. The class $\mathcal{C}$ will be assumed to be full in the sense that $\mathcal{C}$ must also be closed under extension of algebras.

Throughout this paper we denote an action of $P$ on $M$ by $p m$, where $P$ and $M$ are k-algebras. We recall the definition of a crossed module, and its pro- $\mathcal{C}$ analogue.

A crossed module 8 is a k-algebra homomorphism $\partial: M \rightarrow P$ together with an action of $P$ on $M$ such that following two Peiffer relations hold (for all $m, m^{\prime} \in M$ and $p \in P)$ :

$$
\begin{array}{ll}
C M 1) & \partial(p m)=p \partial(m), \\
C M 2) & \partial(m) m^{\prime}=m m^{\prime} .
\end{array}
$$

We denote such a crossed module by $(M, P, \partial)$.

If $(M, P, \partial)$ and $\left(M^{\prime}, P^{\prime}, \partial^{\prime}\right)$ are two crossed modules, a crossed module morphism $(\phi, \psi):(M, P, \partial) \rightarrow\left(M^{\prime}, P^{\prime}, \partial^{\prime}\right)$ is a tuple which consists of k-algebra homomorphisms, $\phi: M \rightarrow M^{\prime}, \psi: P \rightarrow P^{\prime}$ such that $\psi \partial=\partial^{\prime} \phi$ and $\phi(p m)=\psi(p) \phi(m)$. Thus we get the category of crossed modules, denoted by XMod .

There are special classes of morphisms, those in which $P=P^{\prime}$ and $\psi$ is the identity morphism. For fixed $P$, a morphism $\left(\phi, i d_{P}\right):(M, P, \partial) \rightarrow\left(M^{\prime}, P, \partial^{\prime}\right)$ will be called a morphism of crossed modules over $P$. Then we have a subcategory $\mathrm{XMod} / P$ of XMod.

Let $(M, P, \partial)$ be a crossed module. $\left(M_{1}, P_{1}, \partial_{1}\right)$ is a subcrossed module of $(M, P, \partial)$ if:

i) $M_{1}$ is a subalgebra of $M$ and $P_{1}$ is a subring of $P$,

ii) $\partial_{1}=\partial_{\mid M_{1}}$, the restriction of $\partial$ to $M_{1}$,

iii) The action of $P_{1}$ on $M_{1}$ is induced by the action of $P$ on $M$.

A subcrossed module $\left(M_{1}, P_{1}, \partial_{1}\right)$ of $(M, P, \partial)$ is a crossed ideal if:

i) $P_{1}$ is an ideal of $P$ and $M_{1}$ is an ideal of $M$,

ii) $p m_{1} \in M_{1}$, for all $p \in P, m_{1} \in M_{1}$,

iii) $p_{1} m \in M_{1}$, for all $p_{1} \in P_{1}, m \in M$.

A pro- $\mathcal{C}$ crossed module $(M, P, \partial)$ is a crossed module in which $M$ and $P$ are pro- $\mathcal{C}$ k-algebras, $\partial$ is a continuous k-algebra homomorphism and the action of $P$ on $M$ is a continuous $P$-action $[6]$.

A morphism of pro- $\mathcal{C}$ crossed modules

$$
(\phi, \psi):(M, P, \partial) \rightarrow\left(M^{\prime}, P^{\prime}, \partial^{\prime}\right)
$$

is a morphism of the underlying crossed modules in which both $\phi$ and $\psi$ are continuous morphisms of pro- $\mathcal{C}$ k-algebras. Thus we get the categories Pro-C.XMod 
and similarly Pro-C.XMod $/ P$ for a fixed codomain $P$; we therefore obtain the forgetful functor:

\section{$\mathcal{U}_{\text {XMod }}:$ Pro-C.XMod $\rightarrow$ XMod.}

Recall from [8] that a cat ${ }^{1}$-algebra is a triple $(E, s, t)$, where $E$ is an k-algebra and $s, t$ are endomorphisms of $E$ satisfying the following conditions:

i) $s t=t$ and $t s=s$

ii) $[$ Kers, Kert $]=0$.

It is well known that there is an equivalence of between the categories XMod and $\operatorname{Cat}^{\mathbf{1}}(\mathbf{A l g})$.

A pro- $\mathcal{C}$ cat ${ }^{1}$-algebra is a cat ${ }^{1}$-algebra $(E, s, t)$ in which $E$ is a pro- $\mathcal{C}$ algebra and $s$ and $t$ are continuous endomorphisms of $E$. A morphism of pro- $\mathcal{C}$ cat $^{1}$-algebra is a morphism

$$
\phi:(E, s, t) \rightarrow\left(E^{\prime}, s^{\prime}, t^{\prime}\right)
$$

of the underlying cat ${ }^{1}$-algebras such that $\phi: E \rightarrow E^{\prime}$ is a continuous morphism of pro- $\mathcal{C}$ algebras. Thus we get the category of pro- $\mathcal{C}$ cat ${ }^{1}$-algebras, denoted by Pro-C.Cat ${ }^{1}(\mathbf{A l g})$. There is a forgetful functor:

$$
\mathcal{U}_{\text {CAlg }}: \text { Pro-C.Cat }{ }^{1}(\mathbf{A l g}) \rightarrow \operatorname{Cat}^{1}(\mathbf{A l g})
$$

It is proven in [6] that, there exists an equivalence of categories Pro-C.XMod and Pro-C.Cat ${ }^{\mathbf{1}}(\mathbf{A l g})$ compatible with the forgetful functors, in the sense of the equivalence between XMod and $\mathbf{C a t}^{\mathbf{1}}(\mathbf{A l g})$.

\section{Crossed Squares and their Pro-C Analogue}

3.1. Crossed squares. The following definition is due to [1].

A crossed square of commutative algebras is a commutative diagram:

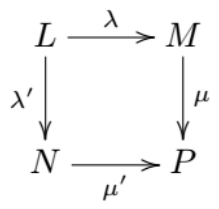

together with actions of $P$ on $L, M$ and $N$ (there are thus actions of $N$ on $L$ and $M$ via $\mu^{\prime}$, and of $M$ on $L$ and $N$ via $\mu$ ) and a function $h: M \times N \rightarrow L$ such that:

1) The maps $\lambda, \lambda^{\prime}, \mu, \mu^{\prime}$ and the composite $\mu \lambda=\mu^{\prime} \lambda^{\prime}$ are crossed modules,

2) The maps $\lambda, \lambda^{\prime}$ preserve the action of $P$,

3) $k h(m, n)=h(k m, n)=h(m, k n)$,

4) $h\left(m+m^{\prime}, n\right)=h(m, n)+h\left(m^{\prime}, n\right)$,

5) $h\left(m, n+n^{\prime}\right)=h(m, n)+h\left(m, n^{\prime}\right)$,

6) $p h(m, n)=h(p m, n)=h(m, p n)$,

7) $\lambda h(m, n)=m \mu^{\prime}(n)$,

8) $\lambda^{\prime} h(m, n)=\mu(m) n$, 
9) $h(\lambda(l), n)=l \mu^{\prime}(n)$,

10) $h\left(m, \lambda^{\prime}(l)\right)=\mu(m) l$

for all $n, n^{\prime} \in N, m, m^{\prime} \in M, p \in P, l \in L, k \in k$. We denote such a crossed square by $(L, M, N, P)$.

Let $\mu$ and $\mu^{\prime}$ are normal subalgebra inclusions and $L=M \cap N$, with $h$ is given by the multiplication in $P$, i.e., $h(m, n)=m n$. Then, we have the crossed square:

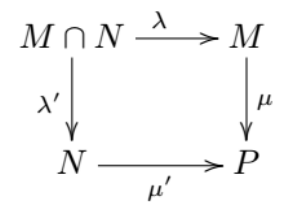

A morphism of crossed square

$$
\Phi=\left(\Phi_{1}, \Phi_{2}, \Phi_{3}, \Phi_{4}\right):\left(L_{1}, M_{1}, N_{1}, P_{1}\right) \rightarrow\left(L_{2}, M_{2}, N_{2}, P_{2}\right),
$$

consists of homomorphisms:

$$
\begin{array}{ll}
\Phi_{1}: L_{1} \rightarrow L_{2}, & \Phi_{2}: M_{1} \rightarrow M_{2}, \\
\Phi_{3}: N_{1} \rightarrow N_{2}, & \Phi_{4}: P_{1} \rightarrow P_{2},
\end{array}
$$

such that the diagram commutes:

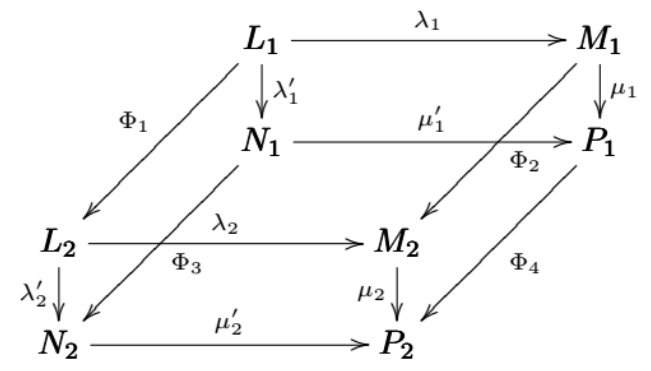

and

$$
\Phi_{1} h\left(m_{1}, n_{1}\right)=h\left(\Phi_{2}\left(m_{1}\right), \Phi_{3}\left(n_{1}\right)\right),
$$

for all $m_{1} \in M_{1}, n_{1} \in N_{1}$, and the homomorphisms $\Phi_{1}, \Phi_{2}, \Phi_{3}$ are $\Phi_{4}$-equivariant.

Thus we get the category of crossed squares, denoted by $\mathrm{Crs}^{2}$.

There are special classes of morphisms, those in which $P_{1}=P_{2}$ and $\Phi_{4}$ is the identity morphism. For a fixed $P$, such a morphism

$$
\Phi=\left(\Phi_{1}, \Phi_{2}, \Phi_{3}, i d\right):\left(L_{1}, M_{1}, N_{1}, P\right) \rightarrow\left(L_{2}, M_{2}, N_{2}, P\right),
$$

will be called a morphism of crossed squares over $P$, yields a subcategory $\mathrm{Crs}^{2} / P$.

A crossed square 


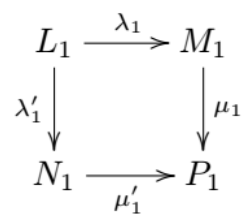

is a subcrossed square of

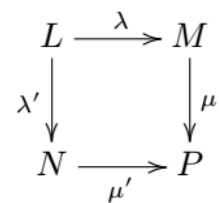

if,

i) $L_{1}$ is a subalgebra of $L, M_{1}$ is a subring of $M, N_{1}$ is a subring of $N, P_{1}$ is a subring of $P$,

ii) $\lambda_{1}$ is restriction of $\lambda$ to $L_{1}, \mu_{1}$ is restriction of $\mu$ to $M_{1}, \lambda_{1}^{\prime}$ is restriction of $\lambda^{\prime}$ to $L_{1}, \mu_{1}^{\prime}$ is restriction of $\mu^{\prime}$ to $N_{1}$,

iii) Actions of $P_{1}$ on $L_{1}, M_{1}$ and $N_{1}$ are induced by the actions of $P$ on $L, M$ and $N$, respectively.

iv) $h_{1}: M_{1} \times N_{1} \rightarrow L_{1}$ is the restriction of $h: M \times N \rightarrow L$ to $M_{1} \times N_{1}$.

A subcrossed square

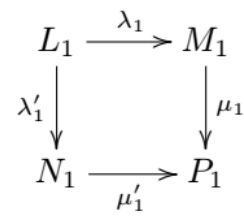

is an ideal of

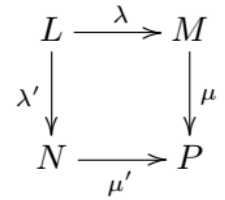

if,

i) $P_{1}$ is an ideal of $P, M_{1}$ is an ideal of $M, N_{1}$ is an ideal of $N$,

ii) For all $p \in P, l_{1} \in L_{1}, m_{1} \in M_{1}$ and $n_{1} \in N_{1}$,

$$
\begin{aligned}
& p l_{1} \in L_{1}, \\
& p m_{1} \in M_{1}, \\
& p n_{1} \in N_{1} .
\end{aligned}
$$


iii) For all $p_{1} \in P_{1}, l \in L, m \in M$ and $n \in N$,

$$
\begin{aligned}
& p_{1} l \in L_{1}, \\
& p_{1} m \in M_{1}, \\
& p_{1} n \in N_{1} .
\end{aligned}
$$

Let

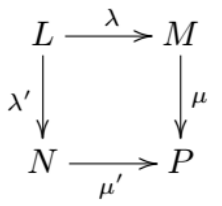

be a crossed square and

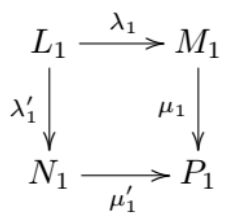

be an ideal of $(L, M, N, P)$. Let $\bar{\lambda}$ is induced by $\lambda, \bar{\mu}$ is induced by $\mu, \overline{\lambda^{\prime}}$ is induced by $\lambda^{\prime}, \overline{\mu^{\prime}}$ is induced by $\mu^{\prime}$. Then there are actions of $P / P_{1}$ on $L / L_{1}, M / M_{1}$ and $N / N_{1}$ given by

$$
\begin{aligned}
& \left(p+P_{1}\right)\left(l+L_{1}\right)=(p l)+L_{1}, \\
& \left(p+P_{1}\right)\left(m+M_{1}\right)=p m+M_{1}, \\
& \left(p+P_{1}\right)\left(n+N_{1}\right)=p n+N_{1}
\end{aligned}
$$

and $N / N_{1}$ on $L / L_{1}$ and $M / M_{1}$ via $\overline{\mu^{\prime}}$, i.e.,

$$
\begin{aligned}
& \left(n+N_{1}\right)\left(l+L_{1}\right)=\mu^{\prime}(n) l+L_{1}, \\
& \left(n+N_{1}\right)\left(m+M_{1}\right)=\mu^{\prime}(n) m+M_{1}
\end{aligned}
$$

and then $M / M_{1}$ acts on $L / L_{1}$ and $N / N_{1}$ via $\bar{\mu}$, i.e.,

$$
\begin{aligned}
& \left(m+M_{1}\right)\left(l+L_{1}\right)=\mu(m) l+L_{1}, \\
& \left(m+M_{1}\right)\left(n+N_{1}\right)=\mu(m) n+N_{1} .
\end{aligned}
$$

for all $p \in P, l \in L, m \in M$ and $n \in N$. The conditions for $\left(L_{1}, M_{1}, N_{1}, P_{1}\right)$ to be ideal in $(L, M, N, P)$ ensure that the actions are well defined. Let $h_{1}: M_{1} \times N_{1} \rightarrow$ $L_{1}$ is defined by $h\left(m+M_{1}, n+N_{1}\right)=h(m, n)+L_{1}$. It is clear that:

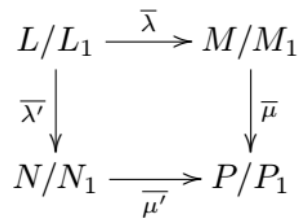

is a crossed square. It is called the quotient crossed square of $(L, M, N, P)$ by $\left(L_{1}, M_{1}, N_{1}, P_{1}\right)$ and denoted by $(L, M, N, P) /\left(L_{1}, M_{1}, N_{1}, P_{1}\right)$. 
3.2. Pro- $\mathcal{C}$ crossed squares. A pro- $\mathcal{C}$ crossed square of algebras

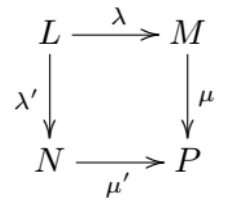

is a crossed square in which $L, M, N$ and $P$ are pro-C algebras, $\lambda, \lambda^{\prime}, \mu, \mu^{\prime}$ are continuous homomorphisms, all the actions are continuous and the $h$-map is continuous.

A morphism

$$
\Phi=\left(\Phi_{1}, \Phi_{2}, \Phi_{3}, \Phi_{4}\right):\left(L_{1}, M_{1}, N_{1}, P_{1}\right) \rightarrow\left(L_{2}, M_{2}, N_{2}, P_{2}\right)
$$

of pro- $\mathcal{C}$ crossed squares is a morphism of the underlying crossed squares in which all the maps $\Phi_{1}, \Phi_{2}, \Phi_{3}$ and $\Phi_{4}$ are continuous morphisms of pro- $\mathcal{C}$ algebras. Thus we get the categories Pro-C.Crs ${ }^{2}$ and similarly Pro-C.Crs ${ }^{2} / P$ for a fixed codomain $P$; and we also get the forgetful functor:

$$
\mathcal{U}_{\mathrm{Crs}^{2}}: \text { Pro-C.Crs }{ }^{2} \rightarrow \mathrm{Crs}^{2} .
$$

Recall the corresponding situation for k-algebras; the forgetful functor

$$
\mathcal{U}_{\text {Alg }}: \text { Pro- } \mathcal{C} . \text { Alg } \rightarrow \text { Alg }
$$

has a left adjoint, known as the pro- $\mathcal{C}$ completion functor, which we will denote by a "ᄉ”.

This is defined as follows:

Let $P$ be a k-algebra and let $\Omega(P)$ be the directed set of finite index ideals $W$ of $P$ with $P / W \in \mathcal{C}$, then

$$
\widehat{P}=\lim _{W \in \Omega(P)} P / W
$$

We will sometimes write $W_{\text {fin }} P$ as indicating that $W \in \Omega(P)$. This notation is useful in as it is more suggestive of the actual concept involved, but can also become somewhat cumbersome so we will use both notations.

We wish to see if the crossed square forgetful functor

$$
\mathcal{U}_{\mathrm{Crs}^{2}}: \text { Pro-C.Crs }{ }^{2} \rightarrow \mathrm{Crs}^{2}
$$

also has a left adjoint. The obvious approach using some idea of finite index ideals is technically messy so we use an equivalence formulation involving Loday's notion of cat ${ }^{2}$-algebras. 


\section{4. $\mathrm{CAT}^{2}$-Algebras and their Pro-C Analogue}

4.1. Cat $^{2}$-algebras. A cat ${ }^{2}$-algebra [1] is a 5 -tuble $\left(E, s_{1}, t_{1}, s_{2}, t_{2}\right)$ where $\left(E, s_{i}, t_{i}\right)$, $i=1,2$ are cat $^{1}$-algebras and

$$
s_{i} s_{j}=s_{j} s_{i}, t_{i} t_{j}=t_{j} t_{i}, s_{i} t_{j}=t_{j} s_{i}
$$

for $i, j=1,2, i \neq j$.

If $\left(E, s_{1}, t_{1}, s_{2}, t_{2}\right)$ and $\left(E^{\prime}, s_{1}^{\prime}, t_{1}^{\prime}, s_{2}^{\prime}, t_{2}^{\prime}\right)$ are cat ${ }^{2}$-algebras a cat ${ }^{2}$-algebra morphism:

$$
\phi:\left(E, s_{1}, t_{1}, s_{2}, t_{2}\right) \rightarrow\left(E^{\prime}, s_{1}^{\prime}, t_{1}^{\prime}, s_{2}^{\prime}, t_{2}^{\prime}\right)
$$

is an algebra homomorphism $\phi: E \rightarrow E^{\prime}$ such that:

$$
\begin{aligned}
& s_{1}^{\prime} \phi=\phi s_{1}, \\
& t_{1}^{\prime} \phi=\phi t_{1}, \\
& s_{2}^{\prime} \phi=\phi s_{2}, \\
& t_{2}^{\prime} \phi=\phi t_{2} .
\end{aligned}
$$

Thus we get the category of cat ${ }^{2}$-algebras, denoted by $\mathbf{C a t}^{2}(\mathbf{A l g})$.

Proposition 1. There is an equivalence of categories between the category of cat ${ }^{2}$ -algebras and that of crossed squares.

Proof. The cat ${ }^{1}$-algebra $\left(E, s_{1}, t_{1}\right)$ will give us a crossed module

$$
\partial: C \rightarrow B
$$

with $C=\operatorname{Ker} s, B=\operatorname{Im} s$ and $\partial=t \mid C$, but as the two cat ${ }^{1}$-algebra structures are independent, $\left(E, s_{2}, t_{2}\right)$ restricts to give cat ${ }^{1}$-algebra structures on $C$ and $B$ and makes $\partial$ a morphism of cat ${ }^{1}$-algebras. Thus we get a morphism of crossed modules

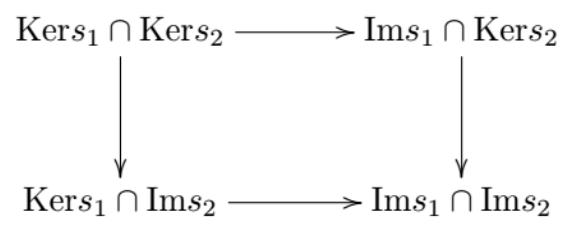

where each morphism is a crossed module for the natural action, i.e. multiplication in $E$. It remains to produce an $h$-map, but it is given by the multiplication within $E$ since if $x \in \operatorname{Ker} s_{2} \cap \operatorname{Im} s_{1}$ and $y \in \operatorname{Im} s_{2} \cap \operatorname{Ker} s_{1}$ then $x y \in \operatorname{Ker} s_{1} \cap \operatorname{Ker} s_{2}$. It is easy to check the crossed squares axioms.

Conversely, if

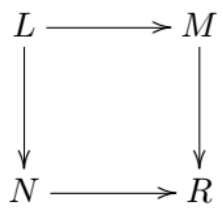


is a crossed square, then we can think of it as a morphism of crossed modules

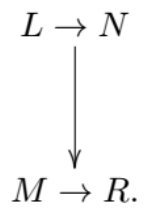

Using the equivalence between crossed modules and cat ${ }^{1}$-algebras this gives a morphism

$$
\partial:(L \rtimes N, s, t) \longrightarrow\left(M \rtimes R, s^{\prime}, t^{\prime}\right)
$$

of cat ${ }^{1}$-algebras. There is an action of $M \rtimes R$ on $L \rtimes N$ given by

$$
(m, r)(l, n)=(r l+\partial(m) l+h(m, n), r n+m n)
$$

for all $(m, r) \in M \rtimes R$ and $(l, n) \in L \rtimes N$. Using this action, we thus form its associated cat ${ }^{1}$-algebra with algebra $(L \rtimes N) \rtimes(M \rtimes R)$ and induced endomorphisms $s_{1}, t_{1}, s_{2}, t_{2}$.

\subsection{Pro- $\mathcal{C}$ cat $^{2}$-algebras.}

Definition 2. A pro-C cat ${ }^{2}$-algebra is a cat ${ }^{2}$-algebra $\left(E, s_{1}, t_{1}, s_{2}, t_{2}\right)$ in which $E$ is a pro-C algebra and $s_{1}, s_{2}, t_{1}$ and $t_{2}$ are continuous endomorphisms of $E$.

A morphism of pro-C cat ${ }^{2}$-algebra

$$
\phi:\left(E, s_{1}, t_{1}, s_{2}, t_{2}\right) \rightarrow\left(E^{\prime}, s_{1}^{\prime}, t_{1}^{\prime}, s_{2}^{\prime}, t_{2}^{\prime}\right)
$$

is a morphism of the underlying cat ${ }^{2}$-algebras such that $\phi$ is a continuous morphism of pro-C algebras. Thus we get the category of pro-C cat ${ }^{2}$-algebras, denoted by Pro $-\mathcal{C} . \operatorname{Cat}^{2}(\mathbf{A l g})$.

There is a forgetful functor:

$$
\mathcal{U}_{\mathrm{C}^{2} \mathrm{Alg}}: \operatorname{Pro}-\mathcal{C} . \mathrm{Cat}^{2}(\mathrm{Alg}) \rightarrow \operatorname{Cat}^{2}(\mathrm{Alg}) .
$$

Theorem 3. There exists an equivalence of categories Pro-C.Crs ${ }^{2}$ and $\mathbf{P r o}-\mathcal{C} . \mathbf{C a t}^{2}(\mathbf{A l g})$ compatible with the forgetful functors, in the sense of the equivalence between $\mathbf{C r s}^{2}$ and $\mathbf{C a t}^{2}(\mathbf{A l g})$, i.e. the following diagram commutes:

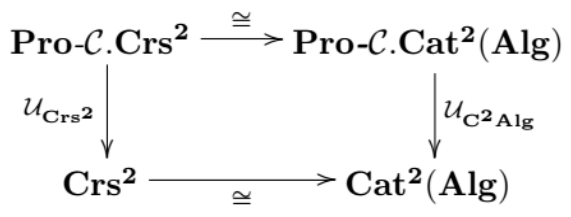

Proof. In fact, if 


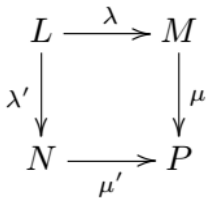

is a pro- $\mathcal{C}$ crossed square, then $E=(L \rtimes M) \rtimes(N \rtimes P)$ is a pro- $\mathcal{C}$ algebra and the endomorphisms $s_{1}, s_{2}, t_{1}$ and $t_{2}$, given before, are continuous, so resulting $\left(E, s_{1}, t_{1}, s_{2}, t_{2}\right)$ is a pro- $\mathcal{C}$ cat ${ }^{2}$-algebra. Similarly if $\left(E, s_{1}, t_{1}, s_{2}, t_{2}\right)$ is a pro- $\mathcal{C}$ cat $^{2}$-algebra then

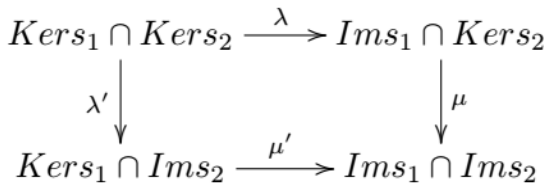

is a pro- $\mathcal{C}$ crossed square.

This lemma will enable us to prove the existence of a left adjoint for

$$
\mathcal{U}_{\mathrm{Crs}^{2}}: \text { Pro-C.Crs }{ }^{2} \longrightarrow \mathrm{Crs}^{2}
$$

by constructing one for

$$
\mathcal{U}_{\mathcal{C}^{2} \mathrm{Alg}}: \text { Pro }-\mathcal{C} \cdot \operatorname{Cat}^{2}(\mathbf{A l g}) \longrightarrow \operatorname{Cat}^{2}(\mathbf{A l g}) .
$$

This latter construction will need projective limit within $\operatorname{Pro}_{-\mathcal{C}} \cdot \mathbf{C a t}^{2}(\mathbf{A l g})$ and so we will briefly look at their construction as it sheds more light on the pro- $\mathcal{C}$ completion functor that will result from their use.

\section{The Completion Process}

Given a projective system $F: I \rightarrow \mathbf{C a t}^{2}(\mathbf{A l g})$, one notes that $F$ is a projective system of algebras together with four endomorphisms of projective systems, $s_{1}, s_{2}, t_{1}, t_{2}: F \rightarrow F$ satisfying

$$
s_{i} s_{j}=s_{j} s_{i}, t_{i} t_{j}=t_{j} t_{i}, s_{i} t_{j}=t_{j} s_{i}
$$

for $i, j=1,2, i \neq j$ and $\left[\operatorname{Kers}_{1}, \operatorname{Kert}_{1}\right]=0,\left[\operatorname{Kers}_{2}, \operatorname{Kert}_{2}\right]=0$. We form $\lim F$ by taking the limit of this underlying system of pro-C algebras together with the induced endomorphism $\lim s$ and $\lim t$. Writing the result as $\left(\bar{F}, \overline{s_{1}}, \overline{t_{1}}, \overline{s_{2}}, \overline{t_{2}}\right)$, we only need to check the conditions $\left[K \operatorname{Ker} \bar{s}_{1}, K e r \overline{t_{1}}\right]=0$ and $\left[K e r \bar{s}_{2}, K e r \overline{t_{2}}\right]=0$. However $\bar{F}$ can be realized as a subalgebra of the product $\prod_{i \in I} F(i)$ and

$$
\overline{s_{1}}\left(\left(x_{i}\right)\right)=\left(s_{1}(i) x_{i}, \overline{t_{1}}\left(x_{i}\right)\right)=\left(t_{1}(i) x_{i}\right),
$$

similarly for $\overline{s_{2}}, \overline{t_{1}}$ and $\overline{t_{2}}$. So the commutator subalgebras $\left[\operatorname{Ker} \overline{s_{1}}, \operatorname{Ker} \overline{t_{1}}\right]$ and $\left[\mathrm{Ker} \overline{\mathrm{s}_{2}}, \mathrm{Ker}_{\overline{t_{2}}}\right.$ are trivial for each $i$ in $I$. 
Proposition 4. A pro-C completion functor from the category $\mathbf{C a t}^{2}(\mathbf{A l g})$ to the category Pro-C.Cat ${ }^{2}(\mathbf{A l g})$ exists, (i.e. the forgetful functor $\mathcal{U}_{\mathcal{C}^{2}} \mathrm{Alg}$ has a left adjoint).

Proof. An exact sequence

$$
0 \longrightarrow\left(E^{\prime}, s_{1}^{\prime}, t_{1}^{\prime}, s_{2}^{\prime}, t_{2}^{\prime}\right) \stackrel{u}{\longrightarrow}\left(E, s_{1}, t_{1}, s_{2}, t_{2}\right) \stackrel{v}{\longrightarrow}\left(E^{\prime \prime}, s_{1}^{\prime \prime}, t_{1}^{\prime \prime}, s_{2}^{\prime \prime}, t_{2}^{\prime \prime}\right) \longrightarrow 0
$$

of cat $^{2}$-algebras is an exact sequence

$$
0 \longrightarrow E^{\prime} \longrightarrow E \longrightarrow E^{\prime \prime} \longrightarrow 0
$$

of the underlying algebras and continuous maps compatible with the source and target maps. In this situation, we say that the cat ${ }^{2}$-algebra $\left(E^{\prime \prime}, s_{1}^{\prime \prime}, t_{1}^{\prime \prime}, s_{2}^{\prime \prime}, t_{2}^{\prime \prime}\right)$ is the quotient of $\left(E, s_{1}, t_{1}, s_{2}, t_{2}\right)$ by the ideal $\left(E^{\prime}, s_{1}^{\prime}, t_{1}^{\prime}, s_{2}^{\prime}, t_{2}^{\prime}\right)$. The latter is of finite index in $\left(E, s_{1}, t_{1}, s_{2}, t_{2}\right)$ if $E^{\prime}$ is finite.

Given any cat ${ }^{2}$-algebra $\left(E, s_{1}, t_{1}, s_{2}, t_{2}\right)$ the set of its ideals $\left(I, s_{1}^{\prime}, t_{1}^{\prime}, s_{2}^{\prime}, t_{2}^{\prime}\right)$ of finite index with $E / I \in \mathcal{C}$ is directed by the inclusion so we can form an inverse system of finite quotient of $\left(E, s_{1}, t_{1}, s_{2}, t_{2}\right)$ and take its limit within the category of pro- $\mathcal{C}$ cat $^{2}$-algebras. (As usual one considers each finite cat ${ }^{2}$-algebra as a pro- $\mathcal{C}$ one having the discrete topology.)

Thus we define a pro- $\mathcal{C}$ completion functor:

$$
\sim: \operatorname{Cat}^{2}(\mathbf{A l g}) \rightarrow \text { Pro-C.Cat }^{2}(\mathbf{A l g})
$$

by

$$
\left(E, \widetilde{s_{1}, t_{1}, s_{2}, t_{2}}\right)=\lim _{\longleftarrow}\left\{\text { finite quotients of }\left(E, s_{1}, t_{1}, s_{2}, t_{2}\right) \text { by }\left(I, s_{1}^{\prime}, t_{1}^{\prime}, s_{2}^{\prime}, t_{2}^{\prime}\right)\right\} \text {. }
$$

Categorically this functor is left adjoint to the forgetful functor from ProC.Cat ${ }^{2}$ (Alg) to $\operatorname{Cat}^{2}(\mathrm{Alg})$.

Proposition 5. A pro-C completion functor from $\mathbf{C r s}^{2}$ to Pro-C.Crs ${ }^{2}$ exists (i.e. the forgetful functor $\mathcal{U}_{\mathbf{C r s}^{2}}$ has a left adjoint).

Proof. In the diagram

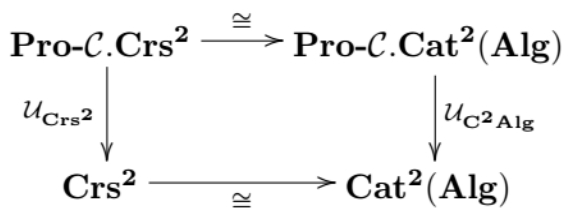

we already found that (11) is the left adjoint functor to right vertical functor. This induces a left adjoint functor to left vertical functor via the equivalence of categories. 
Remark 6. One can attempt to use the functors defining the two equivalence to give an "explicit" description of this pro-C completion functor, but in what follows we shall merely use its existence and the universal property that it satisfies to compare it with the individual algebras involved.

Notation 7. We denote the pro-C completion of the crossed square $(\widetilde{(L, M, N, P})$ or less accurately, $(\widetilde{L}, \widetilde{M}, \widetilde{N}, \widetilde{P}))$, as follows:
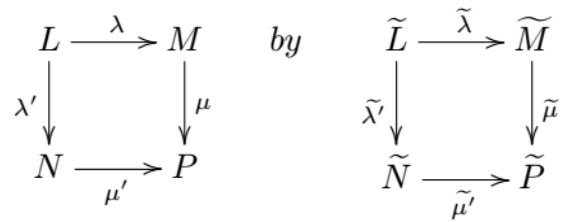

It is natural to compare this pro- $\mathcal{C}$ completion $(\widetilde{L}, \widetilde{M}, \widetilde{N}, \widetilde{P})$ with the pro- $\mathcal{C}$ completions $\widehat{L}, \widehat{M}, \widehat{N}, \widehat{P}$ and $\widehat{\lambda}, \widehat{\mu}, \widehat{\lambda^{\prime}}, \widehat{\mu^{\prime}}$ of the individual pieces of data involved. One may even wonder why

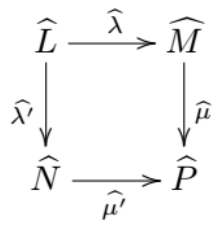

is not itself always the same as,

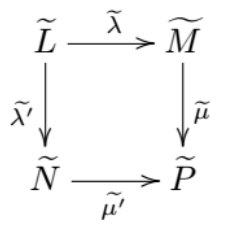

To start the study of this problem we first look at $\widetilde{P}$.

Proposition 8. For any crossed square $(L, M, N, P)$, we have $\widetilde{P} \cong \widehat{P}$.

Proof. This follows from an adjoint functor argument: There is a forgetful functor

$$
R: \mathrm{Crs}^{2} \rightarrow \mathrm{Alg}
$$

given by $R(L, M, N, P)=P$ also an analogous one

$$
R_{p \mathcal{C}}: \text { Pro-C.Crs }{ }^{2} \rightarrow \text { Pro-C.Alg. }
$$

These have left adjoints $L$ and $L_{p \mathcal{C}}$ defined by $L(P)=(P, P, P, P)$ 


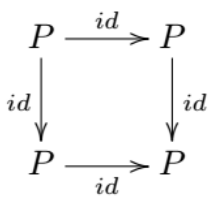

with the $h$-map given by $h\left(p, p^{\prime}\right)=p p^{\prime}$ for all $p, p^{\prime} \in P$ and similarly for $L_{p \mathcal{C}}$.

Then, we get the diagram:

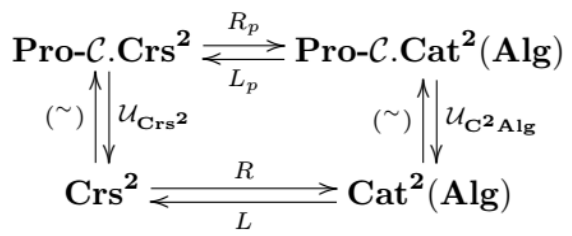

The right adjoint diagram commutes, so the left adjoint diagram commutes up to isomorphism, i.e.

$$
(\widetilde{P, P, P, P}) \simeq(\widehat{P}, \widehat{P}, \widehat{P}, \widehat{P})
$$

but better we have a sequence of isomorphisms: for a pro- $\mathcal{C}$ algebra $H$,

$$
\begin{aligned}
& \text { Pro-C.Alg }\left(R_{p \mathcal{C}}(L, \widetilde{M, N}, P), H\right) \\
& \cong \operatorname{Pro}^{2} \text {. } . \text { Crs }^{2}\left((L, \widetilde{M, N}, P), L_{p \mathcal{C}}(H)\right) \\
& \cong \operatorname{Crs}^{2}\left((L, M, N, P), U_{\mathbf{C r s}^{2}} L_{p \mathcal{C}}(H)\right) \\
& \cong \operatorname{Crs}^{2}\left((L, M, N, P), L U_{\mathcal{C}^{2}} \mathrm{Alg}(H)\right) \text { by observation } \\
& \cong \mathbf{A} \lg \left(R(L, M, N, P), U_{\mathbf{A l g}}(H)\right) \\
& \cong \operatorname{Alg}\left(P, U_{\mathbf{A l g}}(H)\right) \\
& \cong \operatorname{Pro}-\mathcal{C} \cdot \operatorname{Alg}(\widehat{P}, H)
\end{aligned}
$$

as required; hence $\widehat{P} \cong \widetilde{P}$, independent from $L, M, N$ are.

In order to study the conditions that yields the isomorphism between $\widetilde{L}, \widetilde{M}, \widetilde{N}$ and $\widehat{L}, \widehat{M}, \widehat{N}$, respectively; we introduce a condition called "cofinality".

Let $(L, P, \partial)$ be a crossed module and write $\Omega_{P}(L)$ for the directed subset of $\Omega(L)$ the set of finite index ideals of $L$, consisting of those $L_{1} \in \Omega(L), L / L_{1} \in \mathcal{C}$, which are $P$-invariant. We will say that $(L, M, N, P)$ satisfies the cofinal condition if $\Omega_{P}(L)$ is cofinal in $\Omega(L)$. It was shown in [6] that if $(L, P, \partial)$ satisfies the cofinality condition, then $\widetilde{L} \cong \widehat{L}$.

Let

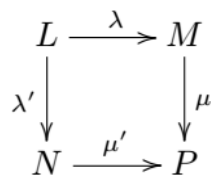


be a crossed square and write $\Omega_{P}(L)$ for the directed subset of $\Omega(L)$ the set of finite index ideals of $L$, given above.

We say that $(L, M, N, P)$ satisfies the cofinal condition if $\Omega_{P}(L)$ is cofinal in $\Omega(L), \Omega_{P}(M)$ is cofinal in $\Omega(M)$ and $\Omega_{P}(N)$ is cofinal in $\Omega(N)$. Note that $\Omega_{P}(L) \subseteq$ $\Omega_{M}(L)$ and $\Omega_{P}(L) \subseteq \Omega_{N}(L)$ so if $\Omega_{P}(L)$ is cofinal in $\Omega(L)$, then $\Omega_{M}(L)$ and $\Omega_{N}(L)$ are cofinal in $\Omega(L)$.

Proposition 9. If $P \in \mathcal{C}$, then any crossed square

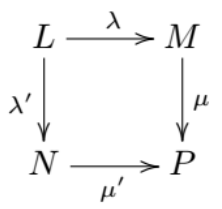

satisfies the cofinality condition.

Proof. Given any $L_{1} \in \Omega(L)$, let

$$
L_{1}^{\prime}=\bigcap_{p \in P} p L_{1}
$$

Then, $L_{1}^{\prime}$ is $P$-invariant and as $P$ in $\mathcal{C}, L_{1}^{\prime}$ is of finite index $L / L_{1}^{\prime} \in \mathcal{C}$. As $L_{1}^{\prime}$ is contained in $L_{1}$, so $\Omega_{P}(L)$ is cofinal in $\Omega(L)$. Similarly, we can show that $\Omega_{P}(M)$ and $\Omega_{P}(N)$ are cofinal in $\Omega(L)$. This completes the proof.

Remark 10. Let $C$ be k-algebra, let $\mathcal{M}(C)$ be the commutative $k$-algebra of multipliers of $C$. Recall that a multiplier of $C$ is a linear mapping $\lambda: C \rightarrow C$ such that for all $c, c^{\prime} \in C ; \lambda\left(c c^{\prime}\right)=\lambda(c) c^{\prime}$, see [2] for more details.

Theorem 11. If $(L, M, N, P)$

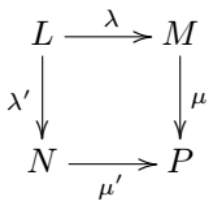

satisfies the cofinality condition, then $\widetilde{L} \cong \widehat{L}, \widetilde{M} \cong \widehat{M}, \widetilde{N} \cong \widehat{N}$.

Proof. Since $\Omega_{P}(M)$ is cofinal in $\Omega(M)$ and $\Omega_{P}(N)$ is cofinal in $\Omega(N), \widehat{M} \cong \widetilde{M}$ and $\widehat{N} \cong \widetilde{N}$. On the other hand since $\Omega_{P}(L)$ is cofinal in $\Omega(L), \widehat{L} \cong \widetilde{L}$.

To check the axioms we need an explicit description of $\widehat{\lambda}: \widehat{L} \rightarrow \widehat{M}, \widehat{\mu}: \widehat{M} \rightarrow \widehat{P}$, $\widehat{\lambda^{\prime}}: \widehat{L} \rightarrow \widehat{N}, \widehat{\mu^{\prime}}: \widehat{N} \rightarrow \widehat{P}, \widehat{\mu \lambda}=\widehat{\mu^{\prime} \lambda^{\prime}}: \widehat{M} \rightarrow \widehat{P}$ and the $h$-map $\widehat{h}: \widehat{M} \times \widehat{N} \rightarrow \widehat{L}$. Given $U_{\text {fin }} P$, there is a composed homomorphism $M \rightarrow P \rightarrow P / U$.

Take $K_{U}$ to be its kernel then since $\mu$ is $P$-equivariant and $P / U$ is finite, it follows that $K_{U}$ is in $\Omega_{P}(M)$ and that $U \subseteq \mathcal{M}_{P}\left(M / K_{U}\right)$. Similarly, there is a 
composed homomorphism $L \rightarrow M \rightarrow M / K_{U}$. Take $T_{U}$ to be its kernel then since $\lambda$ is $M$-equivariant and $P / U$ is finite, it follows that $T_{U}$ is in $\Omega_{M}(L)$ and that $K_{U} \subseteq \mathcal{M}_{P}\left(L / T_{U}\right)$. On the other hand there is also composed homomorphism $L \rightarrow$ $P \rightarrow P / U$.

Take $H_{U}$ to be its kernel then since $\mu \lambda$ is $P$-equivariant and $P / U$ is finite, it follows that $H_{U}$ is in $\Omega_{P}(L)$ and that $U \subseteq \mathcal{M}_{P}\left(L / H_{U}\right)$. Similarly there are composed homomorphisms $N \rightarrow P \rightarrow P / U, L \rightarrow N \rightarrow N / K_{U}^{\prime}$ and $L \rightarrow P \rightarrow P / U$ and kernels $K_{U}^{\prime}, T_{U}^{\prime}, H_{U}^{\prime}$ of these morphisms respectively. It is easy to show that $H_{U}=T_{U}=T_{U}^{\prime}=H_{U}^{\prime}$. These observations readily imply that $\widehat{\lambda}, \widehat{\mu}, \widehat{\lambda^{\prime}}, \widehat{\mu^{\prime}}, \widehat{\mu \lambda}=\widehat{\mu^{\prime} \lambda^{\prime}}$ and the $h$-map $\widehat{h}$, defined by

$$
\begin{array}{ll}
\widehat{\lambda}_{U}\left(l T_{U}\right)=\lambda l_{U} K_{U}, & \widehat{\mu}_{U}\left(m K_{U}\right)=\mu m_{U} U, \\
{\widehat{\lambda^{\prime}}}_{U}\left(l T_{U}\right)=\lambda^{\prime} l_{U} K_{U}^{\prime}, & {\widehat{\mu^{\prime}}}_{U}\left(n K_{U}^{\prime}\right)=\mu n_{U} U, \\
\widehat{\mu \lambda}_{U}\left(l H_{U}\right)=(\mu \lambda) l_{U} U, & \widehat{\mu^{\prime} \lambda^{\prime}}\left(l H_{U}\right)=\left(\mu^{\prime} \lambda^{\prime}\right) l_{U} U, \\
\widehat{h}_{U}\left(m K_{U}, n K_{U}^{\prime}\right)=h(m, n)_{U} H_{U} . &
\end{array}
$$

It is clear that $\widehat{\mu \lambda}_{U}=\widehat{\mu}_{U} \widehat{\lambda}_{U}={\widehat{\mu^{\prime}}}_{U}{\widehat{\lambda^{\prime}}}_{U}={\widehat{\mu^{\prime} \lambda^{\prime}}}_{U}$. Rest of the proof follows from the crossed square axioms of $(L, M, N, P)$ and the descriptions of $\widehat{\lambda}, \widehat{\mu}, \widehat{\lambda^{\prime}}, \widehat{\mu^{\prime}}, \widehat{\mu \lambda}=\widehat{\mu^{\prime} \lambda^{\prime}}$, $\widehat{h}$ and the $\widehat{P}$-action.

Corollary 12. If $P$ is in $\mathcal{C}$, and

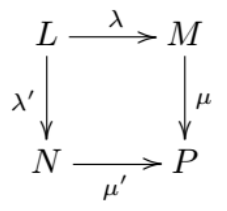

is a crossed square then

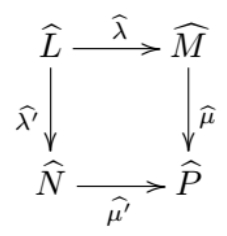

is a crossed square, which is the pro-C completion of $(L, M, N, P)$.

\section{REFERENCES}

[1] Arvasi, Z., Crossed squares and 2-crossed modules of commutative algebras, Theory and Applications of Categories, 3(7), (1997), 160-181. 
[2] Arvasi, Z. and Ege, U., Annihilators, multipliers and crossed modules, Applied Categorical Structures, 11, (2003), 487-506.

[3] Aytekin, A. and Koçak, M., Pro-C completions process of crossed squares and some relations, World Applied Sciences Journal, 7 (7), (2009), 856-865.

[4] Ellis, G.J., Higher dimensional crossed modules of algebras, J. Pure and Appl. Algebra, 52, (1988), 277-282.

[5] Ellis, G.J., Crossed modules and their higher dimensional analogues, University of Wales, Ph.D. Thesis, (1984).

[6] Koçak, M., Pro-C completions of crossed modules of commutative algebras, Algebras, Groups and Geometries, Vol 22(2), (2005).

[7] Kokers, K.J. and Porter, T., Pro-C Completions of crossed modules, Proceedings of the Edinburgh Mathematical Society, 33-1 (1990), 39-51.

[8] Porter, T., Homology of commutative algebras and an invariant of Simis and Vasconceles, J.Algebra, 99, 2, (1987).

[9] Ribes, L. and Zalesskii, P., Profinite groups, Ergebnisse der Mathematik und ihrer Grenzgebiete 3. Folge, Springer-Verlag, Berlin, (2000).

[10] Whitehead, J.H.C., Combinatorial homotopy II., Bull. Amer. Math. Soc., 55, (1949), 213245.

Current address: Hatice Gülsün Akay: Department of Mathematics and Computer Science, Eskişehir Osmangazi University, Turkey.

E-mail address: hgulsun@ogu.edu.tr

ORCID Address: http://orcid.org/0000-0001-7983-6852 